Direct Fabrication of Unsupported Inclined Aluminum Pillars based on Uniform Micro Droplets Deposition

\author{
Daicong Zhang ${ }^{1}$, Lehua $\mathrm{Qi}^{1,2^{*}}$, Jun Luo ${ }^{1,2}$, Hao $\mathrm{Yi}^{1}$, Xianghui Hou ${ }^{3}$ \\ ${ }^{1}$ School of Mechanical Engineering, Northwestern Polytechnical University, Xi'an 710072, \\ China. \\ ${ }^{2}$ Education Ministry Key Laboratory of Modern Design and Integrated Manufacturing \\ Technology, Northwestern Polytechnical University, Xi'an 710072, China \\ ${ }^{3}$ Faculty of Engineering, University of Nottingham, Nottingham NG7 2RD, United Kingdom.
}

\begin{abstract}
In order to investigate forming directly complex parts without support materials or structures by uniform micro droplets deposition technique, the present work focus on fabricating the unsupported inclined aluminum pillars through offset deposition. An experimental system is developed to produce and deposit uniform molten aluminum droplets. A model is introduced to describe the inclined angle of the droplet deposition at different offset ratios. A one dimensional heat transfer model is proposed to help select the initial temperature parameters of the impinging droplet and the previous solidified droplet to ensure that the fusion occurs. No melting, partial melting and excessive melting region at different offset ratios are determined. The correspondence between offset ratio and inclined angle is considered to be a simple cosine function, and the hypothesis is verified by experiments. The influence of deposition error on an inclined angle of pillars is studied. Internal microstructure of droplet fusion is observed in order to ensure good metallurgical bonding. All of these studies show the feasibility of fabricating directly unsupported inclined aluminum pillars in the limited angle range by using uniform micro droplets.
\end{abstract}

Keywords: inclined aluminum pillars; fusion ratio; offset ratio; micro droplets deposition

\title{
1.1. Introduction
}

Uniform molten metal droplets technique draws more and more attention due to its potential of fabricating rapidly metallic components [1-3]. In this technique, 3D object can be fabricated through a highly flexible "digital fabrication" process [4, 5]. At present, these parts are mostly two-dimensional structure [6, 7]. Complex parts are formed mainly by the powder [8] or using removable structure [9] as supports in additive manufacturing. However, when a part has micro 
slender pipe $[10,11]$ or porous features [12], it will be difficult to clear the support materials or structures. Therefore, it is necessary to develop a new direct forming method to fabricate complex structure. Characteristics of offset deposition between micro droplets can be to provide a potential method without support $[2,13,14]$. It provides a possibility for fabricating directly micro complicated metal structures, because the metal droplet diameter can be controlled in the range of $100 \sim 1000 \mu \mathrm{m}[2,6,7,14]$.

The key problem is to control spreading and fusion shape of deposited droplets at different offset ratios. Droplet fusion is a complicated fluid and thermal behavior, which includes impacting [15], spreading [16], re-melting [17], oscillation [18] and solidification [19] of metal droplets. Low Weber number deposition is a prerequisite to maintain the droplet spreading controllable [18]. Impinging velocities as an important parameter of Weber number strongly affect the final shapes of the pileup [20]. More complex inclined impact of a molten droplet deposited onto a substrate is predicted usually by numerical model [21, 22]. The impact fluid dynamics, cooling and subsequent solidification of the impinging droplet in the pile-up is strongly influenced by the geometry of the previous solidified droplet [20]. In addition, the final spreading shape depends mainly on spreading time and solidification time. A necessary condition, though not sufficient, for a droplet to take on a final spherical cap shape is that the liquid comes to rest before significant solidification takes place [18]. Good fusion is important for forming quality. To ensure good fusion, droplet temperature and substrate temperature need to be selected properly $[14,23]$. Re-melting between impinging droplet and previous droplet could be considered as an one-dimensional Stefan problem with phase change [14, 19, 24], where the thermal and dynamic behaviors of molten metal droplets belong to non-isothermal deposition [25]. Less re-melting and poor metallurgical bonding under inappropriate droplet temperature or substrate temperature will result in micro-void and cold lap [5, 26].

The aim of the present work is to investigate fabricating directly unsupported inclined aluminum pillars by molten aluminum droplets under different fusion ratios and offset ratios. An experimental system of ejecting and depositing uniform molten aluminum alloy droplets is developed. A model is introduced to describe the inclined angle of the droplet deposition at different offset ratios. A one dimensional heat transfer model is proposed to select the initial temperature parameters of impinging droplet and previous solidified droplet to ensure that fusion occurs. The correspondence between offset ratio and inclined angle is verified by experiments. Internal microstructure of droplet fusion is observed in order to ensure good 
metallurgical bonding. The potential feasibility of producing complex 3D metal parts deposited by molten metal droplets has been demonstrated.

\subsection{Experimental system and method}

Schematic diagram of the experimental apparatus is shown in Fig. 1a, which consists mainly of a three-dimensional platform, a metal melting crucible, a protective gas system, a pulse generation system, a temperature detection system, and a substrate heating system. Numerical Control (NC) code generated by host computer is downloaded into Programmable Multi Axis Controller (PMAC), which controls three-dimensional platform to the corresponding positions, then triggers piezoelectric ceramic. The stress wave is produced by micro vibration of the bar in the metal liquid. At the peak of the stress wave, a little liquid is squeezed out of the nozzle. At the trough of the stress wave, the liquid near the nozzle is retracted and separated from the liquid out of the nozzle. Micro metal droplets are ejected onto the substrate by the micro-sized nozzle. At the moment of colliding with substrate, a hemispherical droplet is formed, the diameter of which is slightly larger than the diameter of the original one. An infrared radiation thermometer is used to measure temperature of the deposited droplets. Then three-dimensional platform is located to the next point position and next metal droplet is ejected. Subsequently, a part is piled up droplet by droplet. 


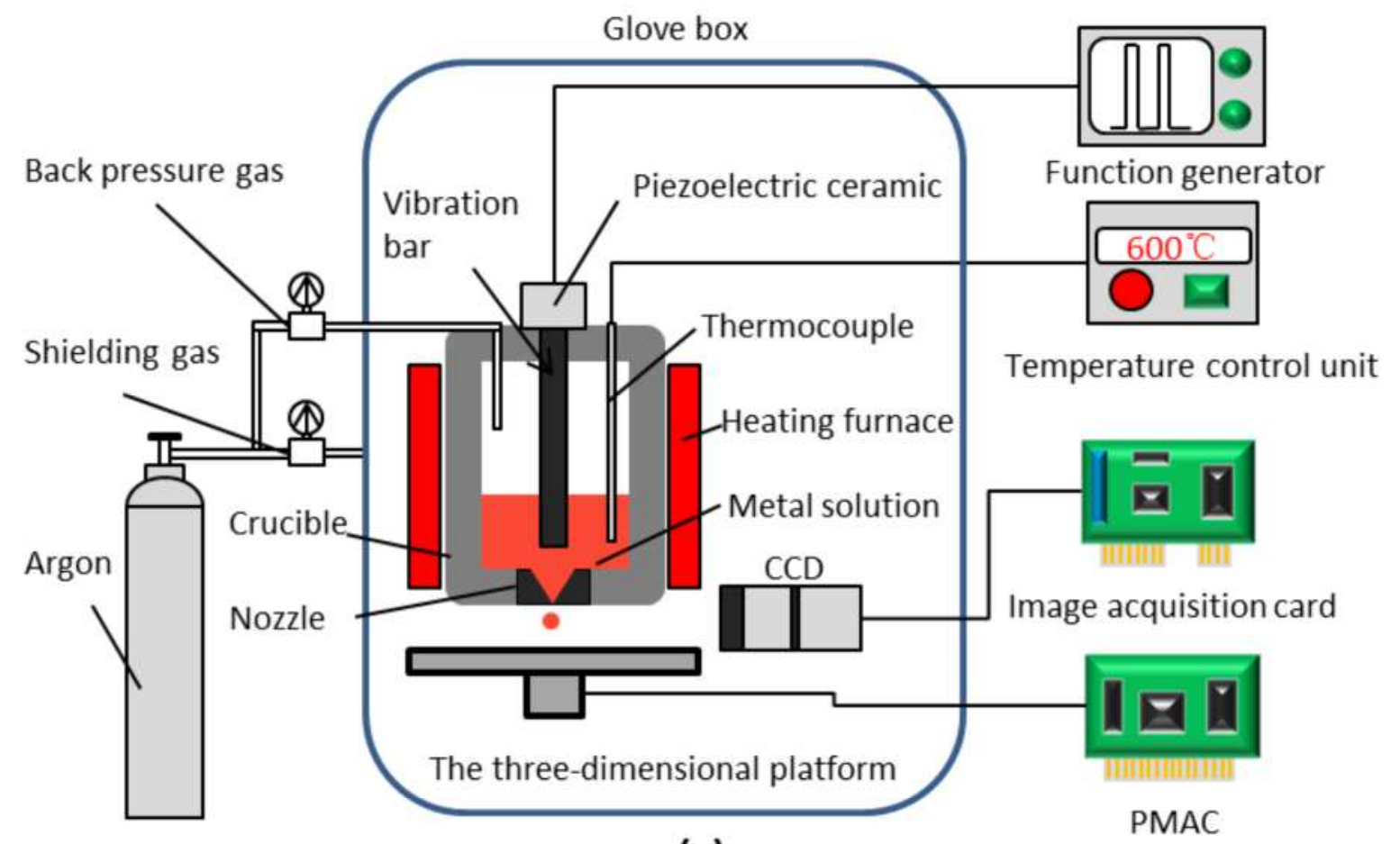

(a)

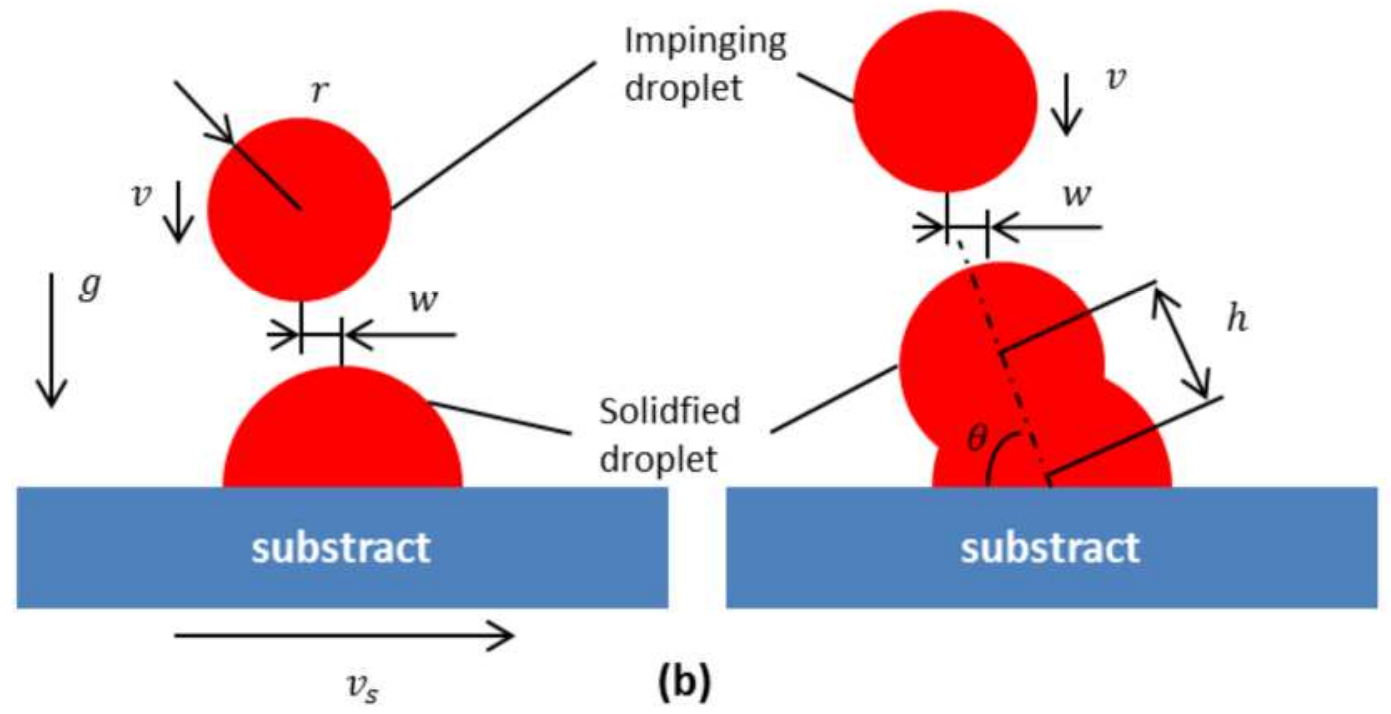

Fig. 1 Uniform micro metal droplet deposition technique. (a) Schematic of the experimental apparatus; (b) Schematic of depositing inclined pillar.

Droplets with initial radius $r$, initial speed $\mathrm{U}$ and trigger frequency $\mathrm{f}$ are ejected to the moving flat substrate. The droplet center-to-center distance $\mathrm{w}$ is adjusted by the moving speed vs of the substrate, which can be expressed as follows:

$$
w=f v_{s}
$$


After the impinging droplet is deposited to the substrate, droplet temperature is gradually reducing to substrate temperature. Then next impinging droplet is non-vertically deposited to the pre-solidified droplet. An inclined pillar with inclined angle $\theta$ is piled up droplet by droplet. Increased height $h$ is the fusion height. The process is shown in Fig. $1 b$.

Aluminum alloy is used as deposition material. The relevant parameters in the experiment are shown in Table 1. Back pressure of the crucible is 3.0 5.0 kPa. Trigger pulse width of the piezoelectric ceramic is $0.5 \sim 1 \mathrm{~ms}$. Distance between the nozzle and the substrate is $5 \mathrm{~mm}$. Oxygen content of the environment in the experimental apparatus is 50 60 PPM (parts per million). Inner diameter of the nozzle is 400,800 and $1000 \mu \mathrm{m}$. Trigger frequency is $2 \sim 5 \mathrm{~Hz}$.

Table 1 The process parameters in the experiment

\begin{tabular}{ll}
\hline Parameter & Value \\
\hline Material & aluminum alloy \\
Back pressure $(\mathrm{kPa})$ & $3.0^{\sim} 5.0$ \\
Pulse width $(\mathrm{ms})$ & $0.5^{\sim} 1$ \\
Deposition distance $(\mathrm{mm})$ & 5 \\
Oxygen content of environment(PPM) & $50 \sim 60$ \\
Nozzle diameter $(\mu \mathrm{m})$ & $400,800,1000$ \\
Trigger frequency $(\mathrm{Hz})$ & $2 \sim 5$ \\
\hline
\end{tabular}

\subsection{Results and Discussion}

Capillarity force and viscous force are hampering the droplet spreading. At low Weber numbers We, the Ohnesorge number can be instead of the Reynolds number, because at low We the spreading process is driven by capillarity forces and is almost unaffected by the impact velocity [18]. We can be calculated by equation (2).

$$
W e=\frac{\rho U^{2} r}{\sigma}
$$

where $\rho$ is droplet density, $U$ is impinging speed, $r$ is the droplet radius and $\sigma$ is surface tension. The Ohnesorge number Oh can be calculated by equation (3) 


$$
O h=\frac{\mu}{\sqrt{\rho \sigma r}}
$$

Table 2 Physical properties of aluminum alloy

\begin{tabular}{ll}
\hline parameter & value \\
\hline Density $\left(\mathrm{kg} / \mathrm{m}^{3}\right)$ & $\rho=2368$ \\
Viscosity $(\mathrm{Pa} \mathrm{s})$ & $\mu=1.257 \times 10^{-3}$ \\
Surface tension $(\mathrm{N} / \mathrm{m})$ & $\sigma=0.868$ \\
Thermal conductivity $(\mathrm{W} /(\mathrm{m} \mathrm{K}))$ & $k_{s}=220, k_{l}=96.4$ \\
Specific heat $(\mathrm{J} /(\mathrm{kg} \mathrm{K}))$ & $C_{p s}=1135, C_{p l}=1086$ \\
Latent heat of fusion $(\mathrm{J} / \mathrm{kg} \mathrm{K})$ & $L=397500$ \\
\hline
\end{tabular}

The impinging speed $U$ is one of the important parameters determining directly the droplet spreading and final shape. A CCD camera system was used to shoot the ejected droplets as shown in Fig. 2a. By calculation, impinging speed of the droplet was about $1.1 \pm 0.05 \mathrm{~m} / \mathrm{s}$. When the inner diameter of the nozzle is $400 \mu \mathrm{m}$, We and $\mathrm{Oh}$ is respectively 0.66 and 0.002 calculated by equation (2) and (3). The effect of impact velocity are negligible [18]. The droplet spreading is driven by the capillarity force at the contact line, and resisted by inertia. So no splash occurs, as shown in Fig. 2 a.

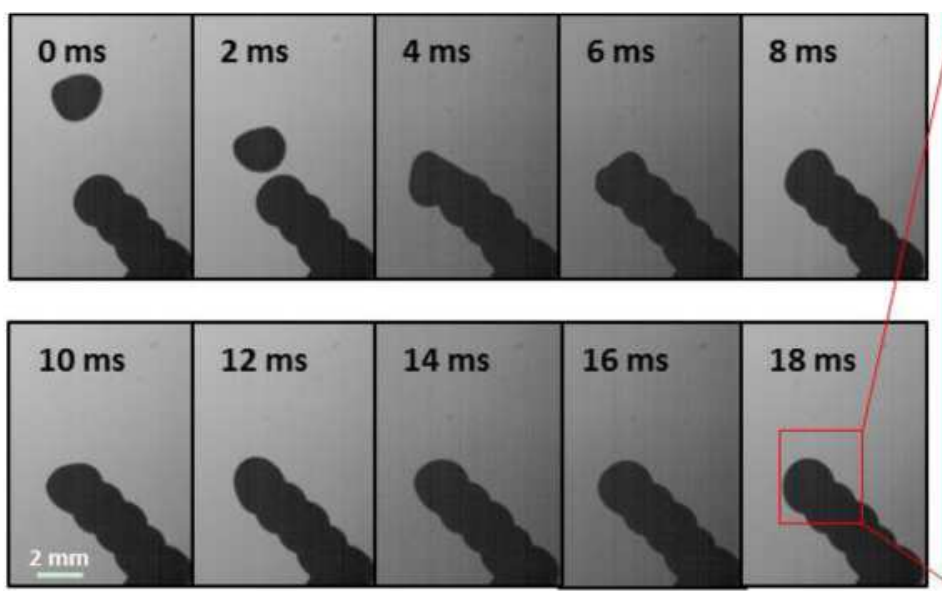

(a)

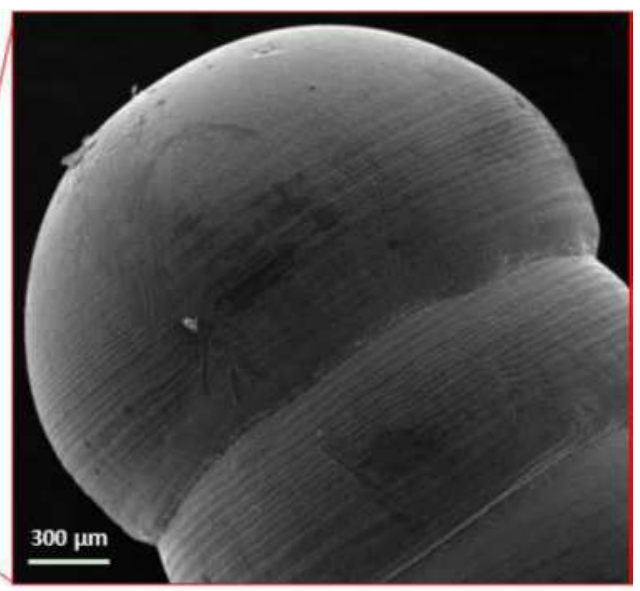

(b) 
Fig. 2 Offset deposition of a single droplet. (a) CCD dynamic graph of the droplet deposition; (b) Scanning electron microscope of the droplet top Initial conditions: $\mathrm{U}=1.1 \pm 0.05 \mathrm{~m} / \mathrm{s}, \mathrm{r}=1.1 \pm 0.03 \mathrm{~mm}, \mathrm{Td}=1023 \mathrm{~K}, \mathrm{Ts}=573 \mathrm{~K}$.

Solidification time ts of a deposited droplet can be calculated estimated by equation (4). The solidification time is much larger than the thermal diffusion time in the droplet and the latent heat effect dominates over the superheat effect of the droplet [18].

$$
\tau_{s} \approx \frac{r^{2}}{\alpha}\left(\frac{L}{c\left(T_{m}-T_{s}\right)}+\frac{T_{d}-T_{m}}{T_{m}-T_{s}}\right)
$$

where, $\alpha$ is thermal diffusivity of the droplet, $\mathrm{c}$ is specific heat of the material, $\mathrm{T}_{\mathrm{d}}$ is temperature of the droplet, Tm is melting point of the material, and $\mathrm{T}_{\mathrm{s}}$ is temperature of the previous solidified droplet.

In Fig. 2, the solidification time $t_{s}$ is about $43 \mathrm{~ms}$ calculated by equation (4). The above estimation is verified by droplet morphology in scanning electron microscope (Fig. 2b). The maximum spreading time is about $10 \mathrm{~ms}$ shoot by the CCD dynamic graph (Fig. 2a). A necessary condition, though not sufficient, for a droplet to form a solid spherical cap is that the liquid comes to rest before significant solidification takes place. The ripple region of the incoming droplet is located from the melting interface to the top, and these ripple gaps are uniform and narrow (Fig. 2b). It also confirms that the oscillation period is very small compared with both the viscous damping time and the solidification time.

The final solid shape of the deposited droplet is approximately a spherical cap, as shown in Fig. 2 , which is helpful for the droplet offset deposition. Spherical cap of the top droplet can fit to offset deposition of next droplet at different offset ratios. Top of next droplet will be also approximately a spherical cap. So a model can be introduced to describe the offset deposition.

First, $\xi$ is defined as an offset ratio, which is the ratio of the droplet center-to-center distance $\mathrm{w}$ and the droplet diameter, as shown in equation (5).

$$
\xi=\frac{w}{2 r}
$$

where $r$ is the droplet radius. 
$\delta$ is defined as fusion ratio, which is the ratio of melting height $\mathrm{h}$ and the droplet diameter, as described in equation (6).

$$
\delta=\frac{h}{2 r}
$$

The inclined angle $\theta$ is calculated by $\mathrm{w}$ and $\mathrm{h}$ [4], as shown in equation (7).

$$
\theta=\arccos \frac{w}{h}
$$

Then equation (7) is simplified into dimensionless form by equation (5) and (6).

$$
\theta=\arccos \frac{\xi}{\delta}
$$

When no melting (Fig. 3d) occurs, melting height $h$ reaches its maximum value, and it is equal to the diameter of the droplet $(\delta=1)$.

$$
h_{\max }=2 r
$$

When ideal melting (Fig. 3b) occurs, two droplets formed a similar cylinder. The cylinder volume $\mathrm{V}_{\text {cylinder }}$ is equal to volume of the droplet $\mathrm{V}_{\text {sphere }}$.

$$
\begin{gathered}
V_{\text {sphere }}=V_{\text {cylinder }} \Rightarrow \frac{4 \pi r^{3}}{3}=h_{\text {ideal }} \pi r^{2} \\
h_{\text {ideal }}=\frac{4 r}{3}
\end{gathered}
$$

Ideal melting height is calculated by equation (10), as shown in equation (11). When melting height is smaller than $h_{\text {ideal, }}$ excessive melting (Fig. 3a) would occur.

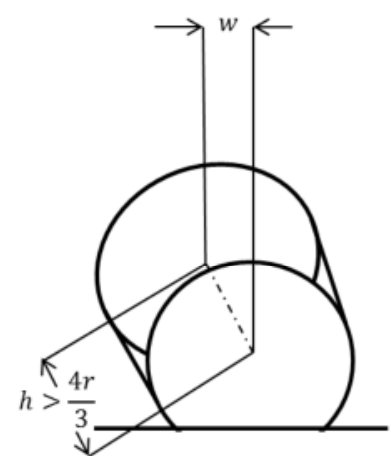

(a)

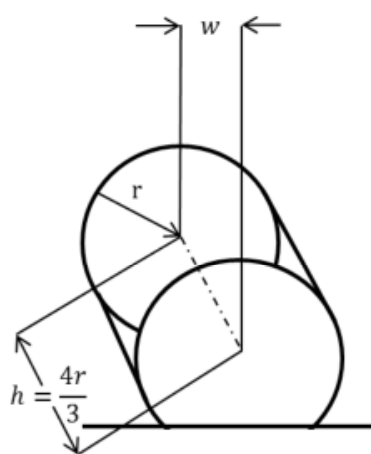

(b)

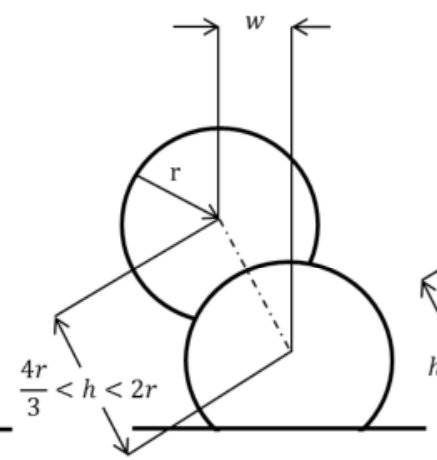

(c)

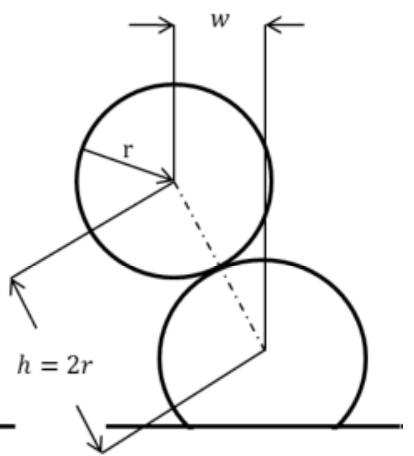

(d) 
Fig. 3 Final fusion shapes at different melting heights. (a) Excessive melting $(\delta<2 / 3)$; (b) Ideal melting $(\delta=2 / 3)$; (c) Partial melting $(2 / 3<\delta<1)$; (d) No melting $(\delta=1)$.

The range of partial melting (Fig. 3c) is determined, as shown in equation (12).

$$
\arccos \frac{w}{h_{\text {ideal }}}<\theta<\arccos \frac{w}{h_{\max }}
$$

Curve about $\xi$ and $\theta$ is drawn through equation (13) calculated by equations (5), (9), (11) and (12).

$$
\arccos \frac{3 \xi}{2}<\theta<\arccos \xi
$$

No melting, partial melting and excessive melting region are defined by equation (13) and shown in Fig. 4. It can be seen that the bigger offset ratio $\xi$ is, the smaller inclined angle $\theta$ is, and the bigger offset ratio $\xi$ is, the more difficult to adjust inclined angle $\theta$ by offset ratio $\xi$.

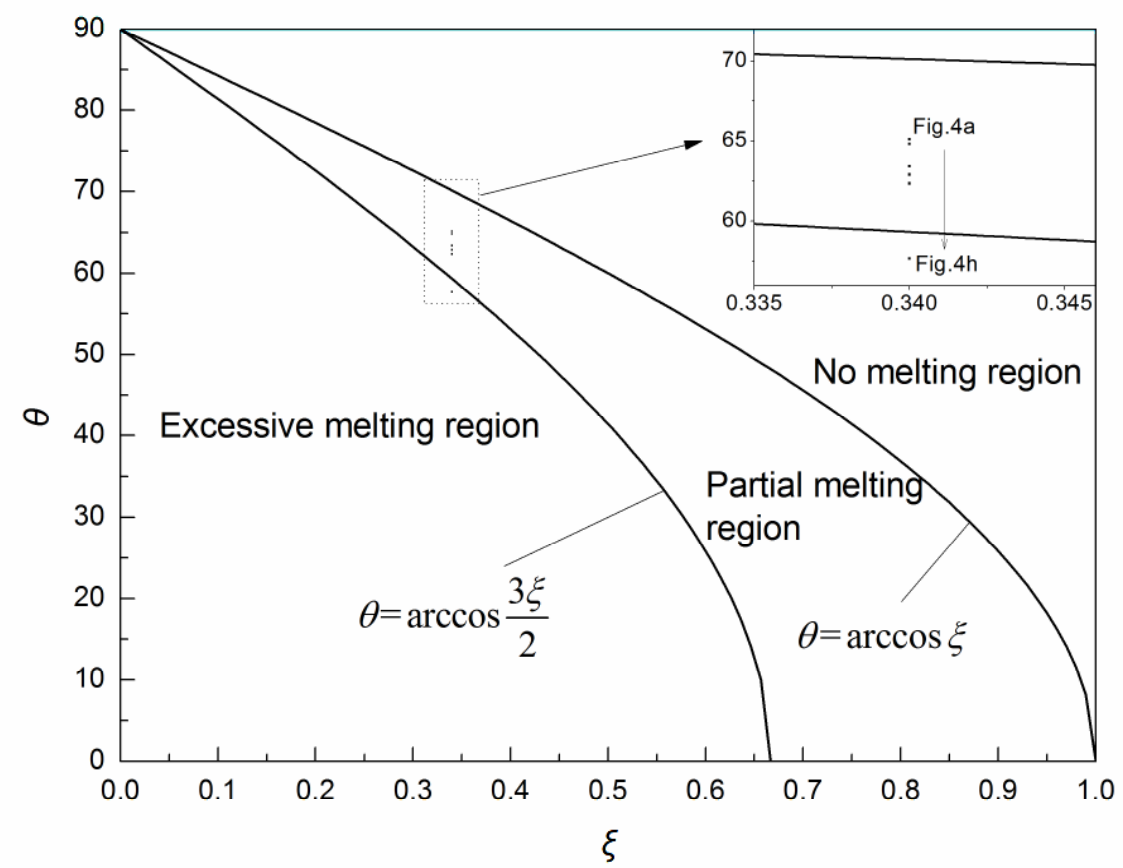

Fig. 4 The impact of fusion depth on inclined angle $\theta\left(^{\circ}\right)$ of pillars at different offset ratios $\xi$

Heat transfer between the impinging droplet and solidified droplet can be modeled as a twophase one dimensional Stefan melting problem [17], as shown in appendix. The position of the melting interface $\mathrm{X}(\mathrm{t})$ is shown in 


$$
X_{(t)}=\left[k_{s} \frac{\left(T_{m}-T_{s}\right) e^{-\lambda^{2}}}{\sqrt{\alpha_{s}} \operatorname{erf}(\lambda)}-k_{l} \frac{\left(T_{l}-T_{m}\right) e^{-\lambda^{2}\left(\alpha_{s} / \alpha_{l}\right)}}{\sqrt{\alpha_{l}} \operatorname{erf}\left(\lambda \sqrt{\alpha_{s} / \alpha_{l}}\right)}\right] \frac{2 \sqrt{t}}{\rho L \sqrt{\pi}}
$$

where $\lambda$ is a parameter related to initial conditions.

When a droplet is deposited on the substrate, the parameter $T_{\mathrm{s}}$ is expressed as the temperature of the substrate. When a droplet is deposited on the solidified droplet, the parameter $T_{\mathrm{s}}$ is expressed as the temperature of the previous droplet. Melting height $\mathrm{h}$ is calculated by equation (15). Melting interface $X_{(t)}$ in equation (15) depends mainly on the droplet temperature $T_{d}$ and the previous solidified temperature $\mathrm{T}_{\mathrm{s}}$.

$$
h=2 r-X_{(t)}
$$

In uniform micro metal droplet deposition technique, the frequency range is usually from zero to several thousand hertz [27]. In the low frequency range, the stable and controllable columnar morphology can be kept in the deposition of inclined pillar $[4,5]$. The low frequency range is confirmed as $0 \sim 23.25 \mathrm{~Hz}$ by equation (16).

$$
f<\frac{l}{t_{s}}
$$

A series of experiments with different droplet temperatures $T_{d}$ and different temperatures of previous solidified droplet $T_{\mathrm{s}}$ had been carried to validate equation (13), as shown in Fig. 4 and Fig. 5. The droplet temperatures $T_{d}$ and substrate temperatures $T_{s}$ can be calculated through the equation (14) to guide the choice of experimental parameters. The experiment proved that when offset ratio $\xi$ is constant, the bigger is the fusion ratio $\delta$, the bigger is the inclined angle $\theta$. 


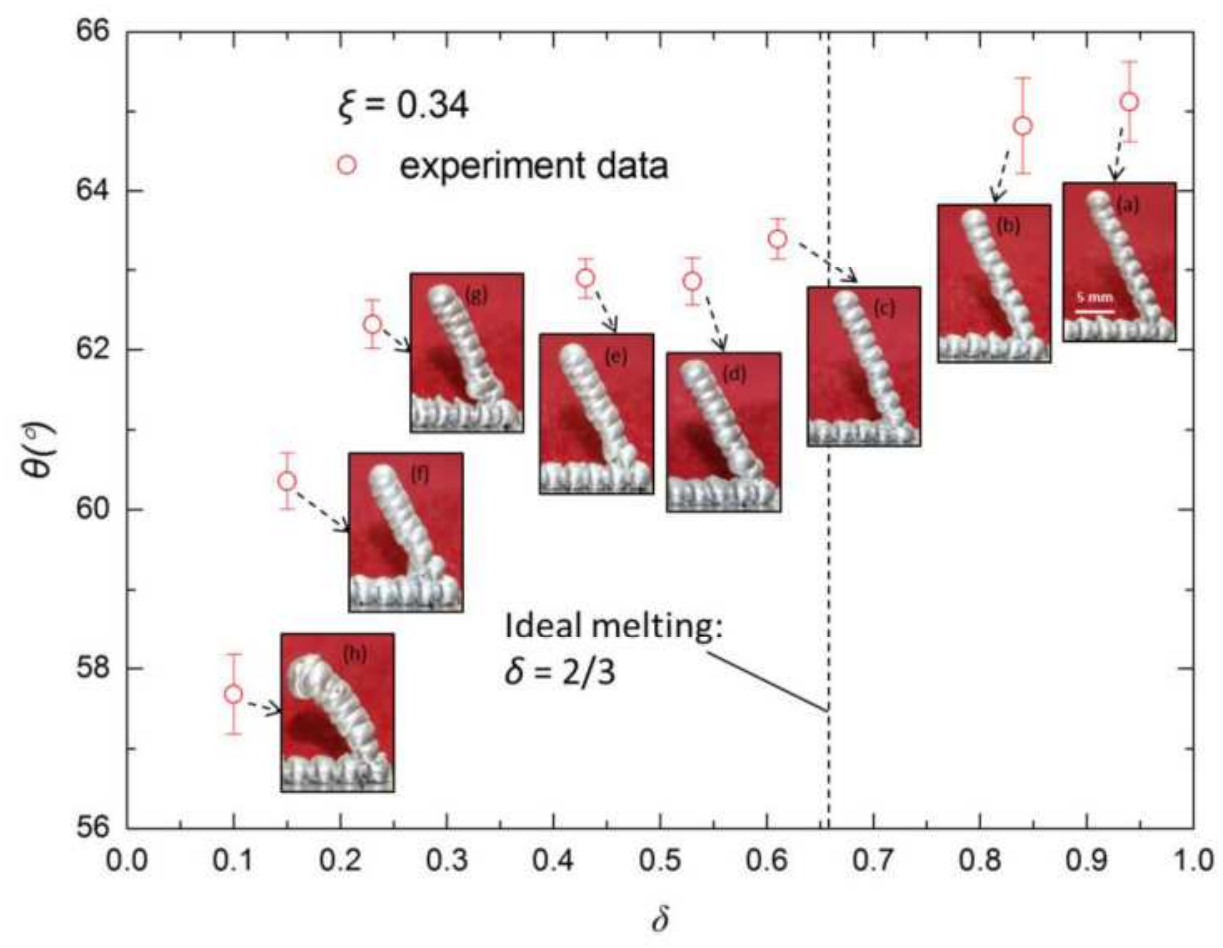

Fig. 5 Inclined pillars with 10 droplets at droplet temperatures $\mathrm{Td}$ and substrate temperatures $\mathrm{T}_{\mathrm{s}}$ of (a) $\mathrm{T}_{\mathrm{d}}=1023 \mathrm{~K}, \mathrm{~T}_{\mathrm{s}}=373 \mathrm{~K}$; (b) $\mathrm{T}_{\mathrm{d}}=1023 \mathrm{~K}, \mathrm{~T}_{\mathrm{s}}=473 \mathrm{~K}$; (c) $\mathrm{T}_{\mathrm{d}}=1023 \mathrm{~K}, \mathrm{~T}_{\mathrm{s}}=573 \mathrm{~K}$; (d) $\mathrm{T}_{\mathrm{d}}=1123 \mathrm{~K}, \mathrm{~T}_{\mathrm{s}}=373 \mathrm{~K}$; (e) $\mathrm{T}_{\mathrm{d}}=1023 \mathrm{~K}, \mathrm{~T}_{\mathrm{s}}=673 \mathrm{~K}$; (f) $\mathrm{T}_{\mathrm{d}}=1123 \mathrm{~K}, \mathrm{~T}_{\mathrm{s}}=473 \mathrm{~K} ;(\mathrm{g}) \mathrm{T}_{\mathrm{d}}=1123 \mathrm{~K}$, $\mathrm{T}_{\mathrm{s}}=573 \mathrm{~K} ;(\mathrm{h}) \mathrm{T}_{\mathrm{d}}=1123 \mathrm{~K}, \mathrm{~T}_{\mathrm{s}}=673 \mathrm{~K}$. Deposition distance and frequency are $5 \mathrm{~mm}$ and $1 \mathrm{~Hz}$ respectively. Initial conditions: $\mathrm{U}=1 \pm 0.02 \mathrm{~m} / \mathrm{s}, \mathrm{r}=1.2 \pm 0.1 \mathrm{~mm}, \xi=0.34$.

Inclined pillars with the angle $\theta$ between $0^{\circ}$ and $90^{\circ}$ were formed by adjusting $\xi$. The inclined angle in theory does not completely agree to experimental data due to the presence of deposition error. The region that the inclined pillar collapses is expressed in Fig. 6 according to deposition error. $\theta$ is calculated by equation (17).

$$
\theta=\arccos \frac{\xi+e}{\delta}
$$

where, e is ratio of deposition error and w.

That is because fusion portion as a support is difficult. When $\xi$ is bigger, support portion is smaller. The fusion ratio $\delta$ is 0.74 . According to $\delta$ and e, the maximum of $\xi$ can be calculated by equation (17), which is 0.67 . 

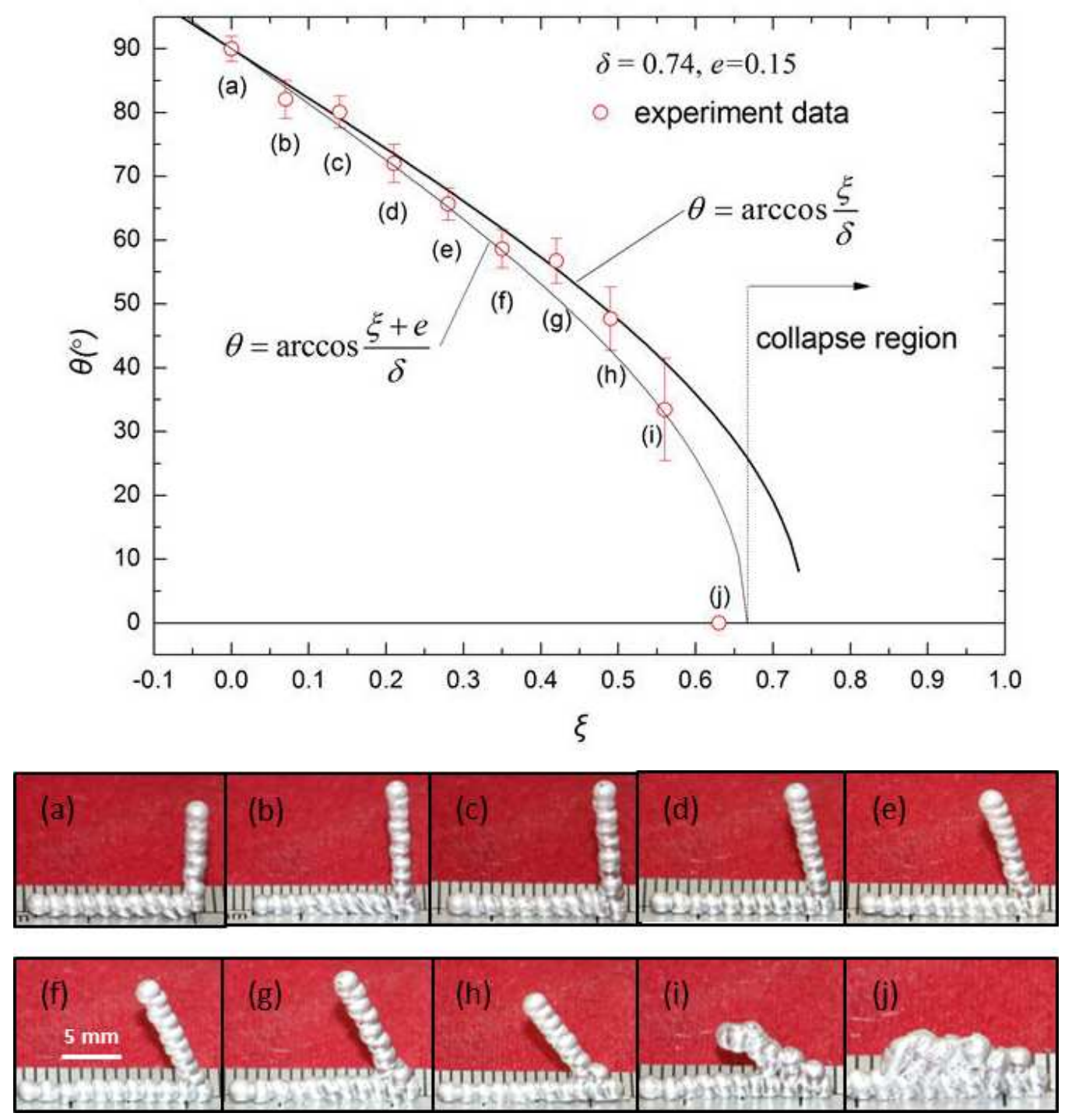

Fig. 6 Inclined pillars with 10 droplets at the initial horizontal center-to-center droplet distance $\mathrm{w}$ of (a) $\mathrm{w}=0$; (b) $\mathrm{w}=0.1 \mathrm{~mm}$; (c) $\mathrm{w}=0.2 \mathrm{~mm}$; (d) $\mathrm{w}=0.3 \mathrm{~mm}$; (e) $\mathrm{w}=0.4 \mathrm{~mm}$; (f) $\mathrm{w}=0.5$ $\mathrm{mm} ;(\mathrm{g}) \mathrm{w}=0.6 \mathrm{~mm}$; (h) $\mathrm{w}=0.7 \mathrm{~mm}$; (i) $\mathrm{w}=0.8 \mathrm{~mm}$; (j) $\mathrm{w}=0.9 \mathrm{~mm}$. Initial conditions: $\mathrm{U}=$ $1 \pm 0.02 \mathrm{~m} / \mathrm{s}, \mathrm{r}=0.9 \pm 0.05 \mathrm{~mm}, \mathrm{~T}_{\mathrm{d}}=1123 \mathrm{~K}, \mathrm{~T}_{\mathrm{s}}=373 \mathrm{~K}, \delta=0.74$.

Deposition direction of the zigzag pattern changes from a direction to another direction in the same plane. In this process, the change of angle slowly occurs, which is different from the regular deposition with a constant angle. Furthermore, the case that the inclined angle slowly changes often takes place in fabricating a complex part. The zigzag pillars were formed to verify whether the spherical cap of the top droplet could fit to offset deposition of next droplet at different offset ratios. Droplets can have very good shape in the corner with variable offset ratio, as shown in Fig. 7. The shape of the deposited droplet is approximately a spherical cap, which is helpful for the droplet offset deposition with variable direction. In Fig. 7(a), offset 
ratios $\xi$ is 0.3 and fusion ratio $\delta$ is 0.82 ; In Fig. 7(b), offset ratios $\xi$ is 0.45 and fusion ratio $\delta$ is 0.71 . These experiments prove the feasibility of changing the forming direction in the inclined column forming at different fusion ratio and offset ratio.
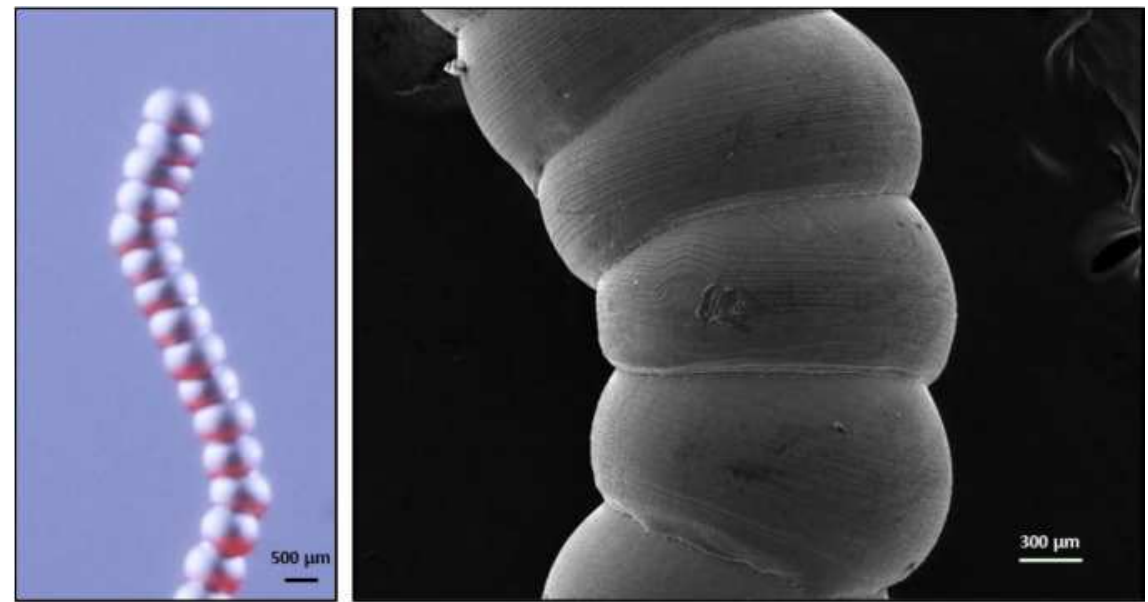

(a)
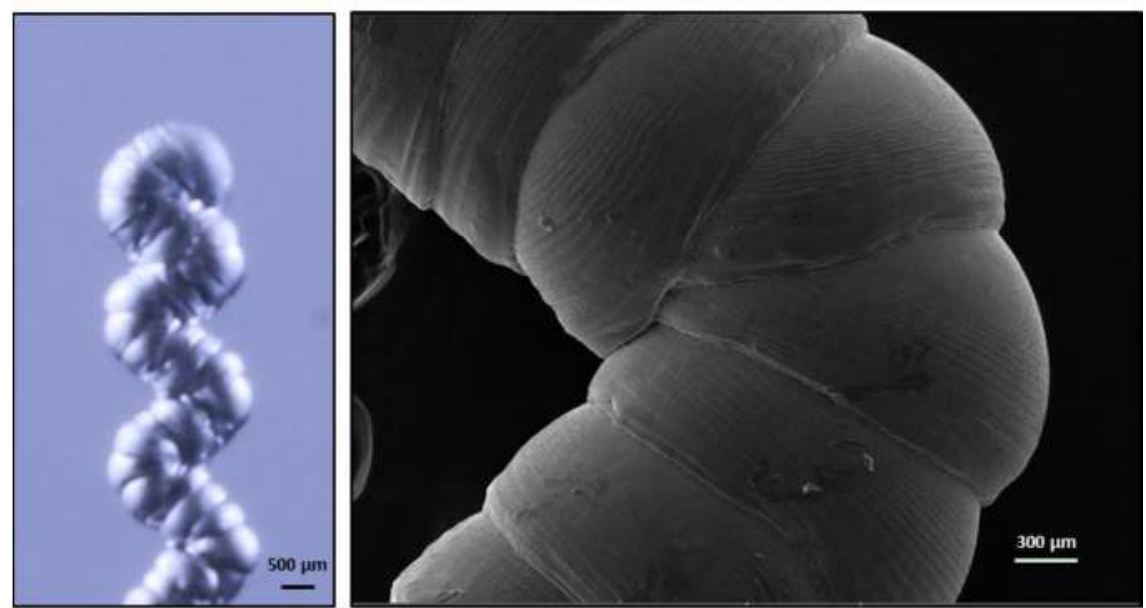

(b)

Fig. 7 Zigzag pillars. (a) $\xi=0.3, \delta=0.82$; (b) $\xi=0.45, \delta=0.71$.

In our previous experiment, the appearance of deposited droplet looks very good, but if the unsuitable parameters are selected, bad metallurgical bonding can be observed by microstructure photograph. Internal microstructure of droplet fusion is shown in Fig. 8. 


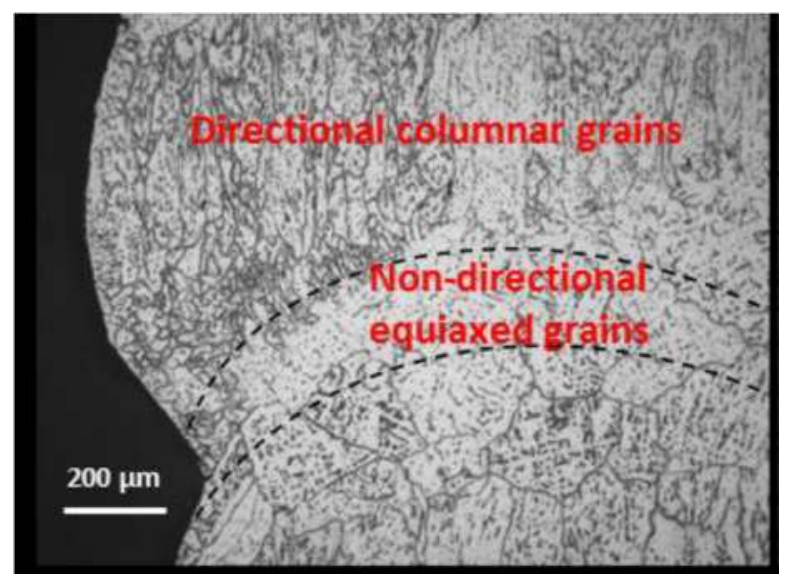

Fig. 8 Internal microstructure of droplet fusion.

As shown in Fig. 8, there are two typical microstructures: the directional columnar grains in the droplet middle zone and the non-directional equiaxed grains in the fusion zone. The predominant microstructure of the solidified droplet is the columnar grains, and only a few equiaxed grains appear in the fusion interface [25]. The fusion interface boundary completely blurred, and where there is full of equiaxed grains. Good metallurgical bonding occurs in the fusion interface.

\section{Conclusions}

(1) Uniform micro aluminum droplet deposition belongs to low Weber number deposition. The spreading process is driven by capillarity forces and is almost unaffected by the impact velocity.

(2) No melting, partial melting and excessive melting region at different offset ratios $\xi$ are determined. In the constant fusion ratio $\delta$, the bigger offset ratio $\xi$ is, the smaller inclined angle $\theta$ is and the more difficult inclined angle $\theta$ is adjusted.

(3) The spherical cap of the top droplet could fit to offset deposition of next droplet from multiple directions. The experiment proved the feasibility of changing the forming direction by forming the zigzag pattern.

(4) Good metallurgical bonding occurs in the fusion interface. There are two typical microstructures: the directional columnar grains in the droplet middle zone and the nondirectional equiaxed grains in the fusion zone. 


\section{Acknowledgements}

This work was supported by National Natural Science Foundation of China (No. 51675436), Natural Science Basic Research Plan in Shanxi Province of China (No. 2015JQ5123), Programme of Introducing Talents of Discipline to Universities (Grant No. B08040).

\section{Appendix Equation Section (Next)}

Heat conduction in the liquid region is described by

$$
\frac{\partial T_{l}}{\partial t}=\alpha_{l} \frac{\partial^{2} T_{l}}{\partial x^{2}} \text { for } 0<x<X_{(t)}, t>0
$$

where $T_{1}$ is equal to $T_{d}, t$ is the time, $x$ is axial distance of the inclined pillar, and $X_{(t)}$ is the position of the melting interface.

Heat conduction in the solid region is described by

$$
\frac{\partial T_{s}}{\partial t}=\alpha_{s} \frac{\partial^{2} T_{s}}{\partial x^{2}} \text { for } 0<x<X_{(t)}, t>0
$$

The solid-liquid interface is assumed to be at the melting point

$$
T(x(t), t)=T_{m} \text { for } t>0
$$

The Stefan condition is applied at the interface

$$
k_{s} \frac{\partial T_{s}}{\partial x}-k_{l} \frac{\partial T_{l}}{\partial x}=L \rho \frac{\partial X}{\partial t} \text { for } x=X_{(t)}, t>0
$$

The initial conditions are

$$
T(x, 0)=T_{s}<T_{m} \text { for } x>0, X_{(0)}=0
$$

The boundary conditions are

$$
\begin{gathered}
T(0, t)=T_{l}>T_{m} \text { for } x>0 \\
T(x, t)=T_{s} \text { for } x \rightarrow \infty, t>0
\end{gathered}
$$

The position of the melting interface $\mathrm{X}_{(\mathrm{t})}$ is shown in 


$$
X_{(t)}=\left[k_{s} \frac{\left(T_{m}-T_{s}\right) e^{-\lambda^{2}}}{\sqrt{\alpha_{s}} \operatorname{erf}(\lambda)}-k_{l} \frac{\left(T_{l}-T_{m}\right) e^{-\lambda^{2}\left(\alpha_{s} / \alpha_{l}\right)}}{\sqrt{\alpha_{l}} \operatorname{erf}\left(\lambda \sqrt{\alpha_{s} / \alpha_{l}}\right)}\right] \frac{2 \sqrt{t}}{\rho L \sqrt{\pi}}
$$

where $\lambda$ is a parameter related to initial conditions.

\section{References}

[1] L.-h. Qi, Y.-p. Chao, J. Luo, J.-m. Zhou, X.-h. Hou, and H.-j. Li, "A novel selection method of scanning step for fabricating metal components based on micro-droplet deposition manufacture," International Journal of Machine Tools and Manufacture, vol. 56, pp. 50-58, 2012.

[2] M. Fang, S. Chandra, and C. B. Park, "Building three-dimensional objects by deposition of molten metal droplets," Rapid Prototyping Journal, vol. 14, pp. 44-52, 2008.

[3] W. Cao and Y. Miyamoto, "Freeform fabrication of aluminum parts by direct deposition of molten aluminum," Journal of Materials Processing Technology, vol. 173, pp. 209-212, 2006.

[4] F. Gao and A. A. Sonin, "Precise deposition of molten microdrops: the physics of digital microfabrication," Proceedings of the Royal Society of London. Series A: Mathematical and Physical Sciences, vol. 444, pp. 533-554, 1994.

[5] Y. P. Chao, L. H. Qi, H. S. Zuo, J. Luo, X. H. Hou, and H. J. Li, "Remelting and bonding of deposited aluminum alloy droplets under different droplet and substrate temperatures in metal droplet deposition manufacture," International Journal of Machine Tools and Manufacture, vol. 69, pp. 38-47, 2013.

[6] X. Yuan, Q. Lehua, C. Yanpu, and L. Jun, "Deposition manufacturing of three-dimensional objects by spraying the metal droplets."

[7] Y.-p. Chao, L.-h. Qi, Y. Xiao, J. Luo, and J.-m. Zhou, "Manufacturing of micro thin-walled metal parts by micro-droplet deposition," Journal of Materials Processing Technology, vol. 212, pp.484-491, 2012.

[8] Y. Hwang, O. H. Paydar, and R. N. Candler, "3D printed molds for non-planar PDMS microfluidic channels," Sensors and Actuators A: Physical, vol. 226, pp. 137-142, 2015.

[9] G. P. Kumar and S. P. Regalla, "Optimization of Support Material and Build Time in Fused Deposition Modeling (FDM)," Applied Mechanics \& Materials, vol. 110-116, pp. 2245-2251, 2011.

[10] D. G. Ahn, S. H. Park, and H. S. Kim, "Manufacture of an injection mould with rapid and uniform cooling characteristics for the fan parts using a DMT process," International Journal of Precision Engineering \& Manufacturing, vol. 11, pp. 915-924, 2010.

[11] E. Sachs, E. Wylonis, S. Allen, M. Cima, and H. Guo, "Production of injection molding tooling with conformal cooling channels using the three dimensional printing process," Polymer Engineering \& Science, vol. 40, pp. 1232-1247, 2000. 
[12] R. Stamp, P. Fox, W. O'Neill, E. Jones, and C. Sutcliffe, "The development of a scanning strategy for the manufacture of porous biomaterials by selective laser melting," Journal of Materials Science: Materials in Medicine, vol. 20, pp. 1839-1848, 2009.

[13] T. M. Lee, T. G. Kang, J. S. Yang, J. D. Jo, K. Y. Kim, B. O. Choi, et al., "3D Metal Microstructure Fabrication using a Molten Metal DoD Inkjet System," in Solid-State Sensors, Actuators and Microsystems Conference, 2007. TRANSDUCERS 2007. International, 2007, pp. $1637-1640$.

[14] M. Fang, S. Chandra, and C. B. Park, "Building vertical walls by deposition of molten metal droplets," in American Society of Mechanical Engineers, Heat Transfer Division, (Publication) HTD, 2005, pp. 325-330.

[15] K. Araki and A. Moriyama, "Theory on Deformation Behavior of a Liquid Droplet Impinging onto Hot Metal Surface," Transactions of the Iron and Steel Institute of Japan, vol. 21, pp. 583$590,1981$.

[16] G. Trapaga, E. F. Matthys, J. J. Valencia, and J. Szekely, "Fluid flow, heat transfer, and solidification of molten metal droplets impinging on substrates: Comparison of numerical and experimental results," Metallurgical \& Materials Transactions B, vol. 23, pp. 701-718, 1992.

[17] J. L. Zarzalejo, S. K. Schmaltz, and H. C. Amon, "Molten droplet solidification and substrate remelting in microcasting Part I: numerical modeling and experimental verification," Heat and Mass Transfer, vol. 34, pp. 477-485, 1999.

[18] S. Schiaffino and A. A. Sonin, "Molten droplet deposition and solidification at low Weber numbers," Physics of Fluids, vol. 9, pp. 3172-3187, 1997.

[19] H. Henry and A. A. Stavros, "Mathematical modelling of solidification and melting: a review," Modelling and Simulation in Materials Science and Engineering, vol. 4, p. 371, 1996.

[20] S. Haferl and D. Poulikakos, "Experimental investigation of the transient impact fluid dynamics and solidification of a molten microdroplet pile-up," International Journal of Heat and Mass Transfer, vol. 46, pp. 535-550, 2003.

[21] D. Tian, Y. Tian, C. Wang, and C. Hang, "Modeling of an oblique impact of solder droplet onto a groove with the impact point to be offset from the groove surfaces interface," Journal of Materials Science, vol. 44, pp. 1772-1779, 2009.

[22] D. W. Tian, Y. H. Tian, C. Q. Wang, and C. J. Hang, "Three-dimensional modelling of solder droplet impact onto a groove," Journal of Physics D: Applied Physics, vol. 41, 2008.

[23] M. Fang, S. Chandra, and C. B. Park, "Experiments on remelting and solidification of molten metal droplets deposited in vertical columns," Journal of Manufacturing Science and Engineering, Transactions of the ASME, vol. 129, pp. 311-318, 2007.

[24] C. Huang and M. Orme, "Phase change manipulation for droplet-based solid freeform fabrication," Transactions of the Asme Serie C Journal of Heat Transfer, vol. 119, pp. págs. 818-823, 1997.

[25] H. S. Zuo, H. J. Li, L. H. Qi, J. Luo, S. Y. Zhong, and Y. F. Wu, "Effect of non-isothermal deposition on surface morphology and microstructure of uniform molten aluminum alloy droplets applied to three-dimensional printing," Applied Physics A: Materials Science and Processing, vol. 118, pp. 327-335, 2014. 
[26] J. Che, S. L. Ceccio, and G. Tryggvason, "Computations of structures formed by the solidification of impinging molten metal drops," Applied Mathematical Modelling, vol. 28, pp. 127-144, 2004.

[27] N. Lass, L. Riegger, R. Zengerle, and P. Koltay, "Enhanced Liquid Metal Micro Droplet Generation by Pneumatic Actuation Based on the StarJet Method," Micromachines, vol. 4, pp. 49-66, 2013. 